\title{
Treatment Outcomes Among Notified Pulmonary Tuberculo- sis Cases in 67 Refugee Health Facilities in the Eastern Afri- ca-IGAD Region: Historical Cohort, 2014-2017
}

\author{
Research Article \\ Volume 2 Issue 2- 2021
}

\begin{abstract}
Author Details
Tsegay legesse ${ }^{1 *}$, Desta Kassa ${ }^{1}$, Fathia Alwan ${ }^{1}$, Dieudonné Tshipala Shindanyi Yiweza ${ }^{2}$, George Sakavarelidze $^{3}$, Marianne Tellier ${ }^{3}$, Goitom Admenur ${ }^{4}$, Dorothy Anjuri ${ }^{5}$, Stephen Muleshe ${ }^{6}$, Stavia Turyahabwe ${ }^{7}$, Nagi Masoud Awad ${ }^{8}$, Evelyn Zbinden ${ }^{3}$, Workneh Gebeyehu ${ }^{1}$

${ }^{1}$ Intergovernmental Authority on Development, Djibouti

${ }^{2}$ UNHCR, Djibouti

${ }^{3}$ Global Fund-Country Team to Ethiopia and IGAD, Ethiopia

${ }^{4}$ Agency for Refugee and Returnees Affair, Ethiopia

${ }^{5}$ Kenya Red Cross Society, Kenya

${ }^{6}$ Ministry of Health, National TB program, Kenya

${ }^{7}$ TB and Leprosy control, Ministry of Health, Kampala, Uganda

${ }^{8}$ National TB program, Federal Ministry of Health, Sudan

*Corresponding author

Tsegay Legesse, Intergovernmental Authority on Development, Djibouti
\end{abstract}

Article History

Received: December 06, 2021 Accepted: December 07, 2021 Published: December 10, 2021

\begin{abstract}
Background: Refugees are at increased risk for Tuberculosis (TB). In 2020, more than 3.9 million refugees were hosted in refugee camps/settlements in member countries of Intergovernmental Authority on Development (IGAD), East Africa. Regional evidence, therefore, on treatment outcome is important to improve the quality of TB care and strengthen the monitoring and evaluation system in the refugee settings.
\end{abstract}

Objective: To assess trends of pooled TB treatment outcomes at the regional level and identify factors associated with unsuccessful treatment outcome among patients with pulmonary TB (PTB), bacteriologically confirmed pulmonary TB (BCPTB) and clinically diagnosed pulmonary TB (CDPTB) in refugee camps in IGAD region from 2014 to 2017.

Methods: A retrospective cohort study was conducted in 67 refugee health facilities located in four countries (Ethiopia=25, Uganda=33, Kenya=5 and Sudan=4). Demographic and clinical data of all PTB patients registered between January 1, 2014 and December 31, 2017 were used. Descriptive analysis was done on the data and multivariate logistic regression analysis was used to identify baseline covariates associated with unsuccessful treatment.

Results: A total of 4,072 TB cases (42\%, 28\%, 12\% and 19\% were from Kenya, Ethiopia, Sudan, and Uganda respectively) were included with more than half $(62.4 \%)$ being BCPTB. $59.2 \%$ were males and $13.5 \%$ were HIV co-infected. The pooled treatment success rate (TSR) in the period under review was $80.7 \%$. TSR was higher among the CDPTB compared to BCPTB cases in 2014 (86.5\% vs. $81.3 \%$ ), 2015 (87.5\% vs. 84.8\%) and 2016 (81.0 vs. 80.6\%); but reversed in favour of BCPTB in 2017 (77.1\% vs. 74.3\%). 0verall, 13.6\% of the cases had unsuccessful treatment (treatment failed, died, or lost to follow-up). The factors significantly associated with unsuccessful treatment were positive HIV status (aOR 2.6[CI: 1.7 - 4.2]), male gender (aOR 1.7[CI: 1.4 - 3.8]), previous TB history (aOR 1.5[CI: 1.0 $2.2])$ and older age (aOR 1.7[CI: $1.1-2.8]$ ).

Conclusion: TB Treatment success rate in the refugee camps remained below the global target ( $\geq 90)$. TB-HIV co-infection, Male gender, previous history of treatment and age (15-34 \&old age) increased the risk for unsuccessful treatment outcomes among PTB patients in the refugee camps.

Keywords: Refugees; Pulmonary tuberculosis; Treatment outcome and east africa-IGAD region 


\section{Background}

Tuberculosis (TB), which caused 10 million people to fall ill (25\% in Africa region) and 1.4 million deaths in 2019, is a leading single infectious agent causing death globally [1]. Despite the great strides on TB control (TB incidence rate declined 1.7\% per year from 2000 to 2019), the world is yet to be on track to achieve the ambitious targets enshrined in the End TB strategy [1]. The global reduction in TB incidence and total number of TB deaths between 2015 and 2019 were $9 \%$ and $14 \%$, respectively, which are less than halfway compared to the End TB 2020 milestones [1]. There are several challenges which may affect the progress to achieving the End TB global milestones. These include missed TB cases, comorbidities (HIV, diabetes), Extensively/ multi-drug resistant TB (XDR/MDR-TB), budget gaps, and TB in high risk populations including refugees $[1,2]$.

Shifting out attention to the latter challenge, we note that refugees and migrants are at particularly high risk for TB incidence and disease progression [3-6], developing MDR-TB [7] and unsuccessful TB treatment outcome [8-10]. This is due to factors including inadequate living conditions, overcrowding, and inadequate TB care and prevention [3-6]. In 2018, there were 70.8 million refugees, asylum seekers and displaced persons in the globe [13]. The InterGovernmental Authority on Development (IGAD), (with Djibouti, Ethiopia, Kenya, Somalia, South Sudan, Sudan and Uganda as the current member countries) is one of the eight regional Economy Communities of the African Union mandated with addressing all human development issues including health, the focus being on youth, children, women, and vulnerable populations including refugees [14].

Because of conflicts and political instabilities experienced in member countries, the region had hosted more than 3.9 million refugees $(56 \%$ of refugees in Africa) in 70 refugee camps/settlements by 2017. Most of them originated from South Sudan, Somalia, Eritrea, Sudan, and Democratic Republic Congo (DRC), with more than 64\% $(2,463,980)$ of them being hosted in four countries (Ethiopia, Uganda, Sudan, and Kenya) [14]. The number of refugees hosted in these four countries continuously increased from 1,389,773 in 2014 to 2,463,980 in 2017.

In addition to the refugee influx, $\mathrm{TB}$ adds to the list of the major problems faced by the IGAD region. This is through the four member countries contributing $20.3 \%$ TB incident cases and $13.9 \%$ of the HIV negative TB mortality estimates in the African region [1].

This calls for the need to track TB treatment success rate (TSR), which is among the ten global priority indicators for achieving the End TB milestones [3] and is used to measure the overall quality and performance of a TB program $[15,16]$. According to WHO End TB global target, at least $90 \%$ treatment success rate (i.e., cured and treatment completed) needed to be achieved by 2020 among people on TB treatment [2]. However, TB treatment success rate among refugees in different parts of the world ranged from $63.6 \%$ to $77.5 \%$ [17-21]. This could be related to the limited evaluation of treatment outcomes in the refugee camps, limited resources [22] or due to the presence of other risk factors that affect the TB treatment outcome $[8,9]$. Previous studies have shown that clinically diagnosed adult pulmonary $\mathrm{TB}$ ( $\mathrm{PTB}$ ) patients, who are perceived to be less contagious to the community, receive lower priority in terms of follow-up and monitoring as compared to bacteriologically confirmed PTB (BCPTB) patients. More so, in Sub-Saharan Africa, where HIV prevalence is high, $[23,24]$ have shown that the number of clinically diagnosed patients has been on the rise and their treatment outcomes are lower than that of BCPTB patients. This suggests the need to analyze TB treatment outcomes among BCPTB and clinically diagnosed PTB (CDPTB) patients in the refugee camps. Overall, TB programs in refugee camps in the IGAD member countries, which are integrated to the general health care system of each country, are supported and led by the National TB Programs (NTPs) of each respective country [2528]. However, there is no specific study which has paid attention to the trends of pooled TB treatment outcome in the refugee health facilities in the IGAD region. This study, therefore, aimed to assess trends of pooled TB treatment outcomes at the regional level and identify factors associated with unsuccessful treatment outcome among patients with PTB, BCPTB and CDPTB patients registered in 67 refugee health facilities in the four IGAD member countries between 2014 and 2017.

\section{Methods}

\section{Study settings and participants}

In 2017, there were 70 refugee camps/settlements in the IGAD region located in Djibouti $(n=3)$, Ethiopia $(n=27)$, Kenya $(n=5)$, South Sudan $(\mathrm{n}=8)$, Sudan $(\mathrm{n}=15)$ and Uganda $(\mathrm{n}=12)$. In these 70 refugee camps, there were 106 health facilities located in Djibouti $(n=2)$, Ethiopia $(\mathrm{n}=28)$, Kenya $(\mathrm{n}=6)$, South Sudan $(\mathrm{n}=8)$, Sudan $(\mathrm{n}=15)$ and Uganda $(n=47)$. Refugee health facilities which provided TB diagnostic and treatment services at least since January 2017 in refugee host countries were included in the study. Thus, a total of 67 refugee health facilities found in four countries (Ethiopia $=25$; Uganda $=33$; Kenya $=5$; Sudan=3) which are in 45 refugee camps (Ethiopia $=25$; Uganda $=12$; Kenya $=5$; Sudan $=4$ ) were included. The list of refugee camps and health faculties included in this study are showed in Supplement 1 The study participants were all PTB patients, categorized as BCPTB and CDPTB, registered for TB treatment in the 67 refugee health facilities between January 1, 2014 and December 31, 2017.

\section{Study design and data collection}

This was a four-year (2014-2017) retrospective cohort study. Demographic and clinical data (age, sex, type of TB, TB patient category, treatment outcome, HIV, and antiretroviral treatment (ART) status) for each study participant were abstracted from TB patient register by trained nurses using a pre-tested data collection tool. Data collection was done from January 2019 to April 2019.

\section{Definitions}

The operational terms used in this study are defined according to the standard definitions in the National TB guidelines of the countries included in this study [25-28] and WHO guideline [29] (Supplement $1)$.

\section{Data management and analysis}

STATA version 13 (Stata Corp, College Station, TX, USA) was used for data checking, cleaning, and analysis. Frequencies, proportions, ratios or mean and standard deviation were calculated to describe demographic, clinical characteristics and treatment outcomes. To identify independent variables associated with unsuccessful treatment outcome, we conducted univariate and multivariable logistic regression model. The independent variables included were age, sex, pre-treatment weight, TB patient category, HIV and ART status, and year of treatment $[11,12]$. Variables with a p-value $<0.05$ in the univariate analysis were included in the multivariable model. Statistical significance level was considered at a p-value $<0.05$.

\section{Results}

\section{Demographic and clinical characteristics of the patients}

The demographic and clinical characteristics of the study participants is shown in Tables 1 \& 2. From 2014 to 2017, a total of 4,072 PTB patients were notified in the 67 refugee health facilities. Fifty-nine percent of the patients were male, and the mean age was 31.5 (SD 18.2) years. The number of notified PTB cases registered for treatment increased 2-fold (742 to 1509) between 2014 and 2017. Majority of the PTB patients were registered in Kenya refugee camps (41.5\%), 
followed by those in Ethiopia (27.6\%), Uganda (19.0\%) and in Sudan (12.0\%). Of the 4,072 PTB patients, 2,539 (62.4\%) were BCPTB and $1,533(37.6 \%)$ CDPTB. The proportion of CDPTB among the PTB patients has decreased from $45.0 \%$ in 2014 to $36.3 \%$ in 2017. Male TB cases were predominant in both $\mathrm{BCPTB}$ and $\mathrm{CDPTB}$ groups, but the overall male cases were higher among BCPTB than CDPTB (62.5\% vs. $53.8 \%)$. Among the CDPTB patients, children ( $<15$ years) constituted $35.4 \%$ while adults aged $15-44$ years constituted $39.3 \%$ of the notified patients. BCPTB patients aged 15-44 years accounted for $71.9 \%$
(Table 1). By patient category, majority of the BCPTB and the CDPTB patients were new cases $(89.3 \%$ vs. $89.6 \%)$, whereas relapse cases were higher among CDPTB than BCPTB (5.4\% vs. $2.9 \%)$ (Table 2). The proportion of HIV positive cases among CDPTB patients increased from $10.5 \%$ in 2014 to $15.4 \%$ in 2017 , while that of BCPTB patients remained in similar across the years of study (Table 2). Over the study period, HIV positive PTB cases who were on ART increased from $72 \%$ in 2014 to $94.2 \%$ in 2017 .

Table 1: Demographic characteristics of the total pulmonary TB (PTB) cases, bacteriologically confirmed PTB (BCPT), and clinically diagnosed PTB (CDPT) patients notified in 67 refugee health facilities in IGAD region, 2014 to $2017(\mathrm{n}=4,072)$.

\begin{tabular}{|c|c|c|c|}
\hline Characteristics & Total PTB $(n=4072)$ N (\%) & BCPT (n=2539) N (\%) & ${ }^{\star} \operatorname{CDPT}(n=1533) N(\%)$ \\
\hline \multicolumn{4}{|c|}{ Gender } \\
\hline Male & $2389(59.2)$ & $1576(62.5)$ & $813(53.8)$ \\
\hline Female & $1644(40.8)$ & $946(37.5)$ & $698(46.2)$ \\
\hline \multicolumn{4}{|c|}{ Age Group } \\
\hline$<15$ & $667(16.4)$ & $116(4.6)$ & $551(35.4)$ \\
\hline $15-24$ & $797(19.6)$ & $627(24.9)$ & $170(11.1)$ \\
\hline $25-34$ & $972(23.9)$ & $731(28.8)$ & $241(15.7)$ \\
\hline $35-44$ & $655(16.1)$ & $461(18.2)$ & $194(12.7)$ \\
\hline $45-54$ & $442(10.9)$ & $298(11.7)$ & $144(9.4)$ \\
\hline $55-64$ & $280(6.9)$ & $176(6.9)$ & $104(6.9)$ \\
\hline$>=65$ & $233(5.7)$ & $115(4.5)$ & $118(7.7)$ \\
\hline Not recorded & $26(0.6)$ & $15(0.6)$ & $11(0.7)$ \\
\hline Mean age $(\mathrm{SD})$, years & $31.5(18.2)$ & $33.9(15.1)$ & $27.7(21.7)$ \\
\hline \multicolumn{4}{|c|}{ Notified Cases in Refugee Health Facilities In } \\
\hline Ethiopia & $1122(27.5)$ & $689(27.1)$ & $433(28.3)$ \\
\hline Kenya & $1691(41.5)$ & $1001(39.4)$ & $690(45.0)$ \\
\hline Sudan & $487(12.0)$ & $237(9.3)$ & $250(16.3)$ \\
\hline Uganda & $772(19.0)$ & $612(24.0)$ & $160(10.4)$ \\
\hline \multicolumn{4}{|c|}{ Notified Cases by Year (Row, \%) } \\
\hline 2014 & $742(100)$ & $408(55.0)$ & $334(45.0)$ \\
\hline 2015 & $840(100)$ & $489(58.2)$ & $351(41.8)$ \\
\hline 2016 & $979(100)$ & $679(69.4)$ & $300(30.6)$ \\
\hline 2017 & $1511(100)$ & $963(63.7)$ & $548(36.3)$ \\
\hline
\end{tabular}

${ }^{*}$ CDPTB: Clinically diagnosed pulmonary tuberculosis, includes smear-negative pulmonary TB and pulmonary smear unknown/not done; SD = Standard deviation.

Table 2: Clinical characteristics of the total pulmonary TB (PTB) cases, bacteriologically confirmed PTB (BCPT), and clinically diagnosed PTB (CDPT) patients notified in 67 refugee health facilities in IGAD region from 2014 to $2017(\mathrm{n}=4072)$.

\begin{tabular}{|c|c|c|c|c|c|c|c|c|c|c|c|}
\hline \multirow{3}{*}{ Characteristics } & \multicolumn{5}{|c|}{ BCPT Patients $(\mathrm{n}=2539)$} & \multicolumn{5}{|c|}{ CDPT Patients $(n=1533)$} & $\begin{array}{c}\text { Total PTB Cases } \\
(2014-2017)\end{array}$ \\
\hline & \multicolumn{5}{|c|}{ N (\%) } & \multicolumn{5}{|c|}{ N (\%) } & \\
\hline & 2014, & 2015, & 2016 & 2017, & Total & 2014 & 2015, & 2016, & 2017 & Total & \\
\hline $\begin{array}{l}\text { PTB cases } \\
\text { notified }\end{array}$ & $\begin{array}{c}407 \\
(16.0)\end{array}$ & $\begin{array}{c}491 \\
(19.3)\end{array}$ & $\begin{array}{c}680 \\
(26.9)\end{array}$ & $\begin{array}{c}961 \\
(37.8)\end{array}$ & $\begin{array}{c}(2014-2017) \\
(\mathrm{n}=2539)\end{array}$ & $\begin{array}{c}334 \\
(21.8)\end{array}$ & $\begin{array}{c}352 \\
(23.0)\end{array}$ & $\begin{array}{c}299 \\
(19.5)\end{array}$ & $\begin{array}{c}548 \\
(35.7)\end{array}$ & $\begin{array}{c}(2014-2017) \\
(\mathrm{n}=1533)\end{array}$ & \\
\hline \multicolumn{12}{|c|}{ Patient Category } \\
\hline New & $\begin{array}{c}360 \\
(88.5)\end{array}$ & $\begin{array}{c}454 \\
(92.5)\end{array}$ & $\begin{array}{c}599 \\
(88.1)\end{array}$ & $\begin{array}{c}855 \\
(89.0)\end{array}$ & $2268(89.3)$ & $\begin{array}{c}308 \\
(92.2)\end{array}$ & $\begin{array}{c}332 \\
(94.3)\end{array}$ & $\begin{array}{c}241 \\
(80.6)\end{array}$ & $\begin{array}{c}492 \\
(89.8)\end{array}$ & $1373(89.6)$ & $3641(89.4)$ \\
\hline Relapse & $23(6.1)$ & $20(4.1)$ & $42(6.2)$ & $\begin{array}{c}53 \\
(5.5)\end{array}$ & $138(5.4)$ & $18(5.3)$ & $4(1.1)$ & $6(2.3)$ & $16(2.9)$ & $44(2.9)$ & $182(4.5)$ \\
\hline $\begin{array}{c}\text { Treatment } \\
\text { after Failure }\end{array}$ & $2(0.5)$ & 0 & $2(0.3)$ & $5(0.5)$ & $9(0.4)$ & $1(0.3)$ & 0 & 0 & 0 & $1(0.1)$ & $10(0.2)$ \\
\hline $\begin{array}{c}\text { LTFU to } \\
\text { Treatment }\end{array}$ & $4(1.0)$ & $4(0.8)$ & $9(1.3)$ & $\begin{array}{c}20 \\
(2.1)\end{array}$ & $37(1.5)$ & $1(0.3)$ & $1(0.3)$ & $6(2.0)$ & $9(1.6)$ & $17(1.1)$ & $54(1.3)$ \\
\hline
\end{tabular}




\begin{tabular}{|c|c|c|c|c|c|c|c|c|c|c|c|}
\hline $\begin{array}{c}\text { Others } \\
\text { previously } \\
\text { treated }\end{array}$ & $1(0.2)$ & $1(0.2)$ & 0 & $3(0.3)$ & $5(0.2)$ & 0 & $4(1.1)$ & $5(1.7)$ & $3(0.5)$ & $12(0.8)$ & $17(0.4)$ \\
\hline Transfer in & $5(1.2)$ & $7(1.4)$ & $24(3.5)$ & $\begin{array}{c}14 \\
(1.5) \\
\end{array}$ & $50(2.0)$ & $4(1.2)$ & $3(0.9)$ & $\begin{array}{c}35 \\
(11.7) \\
\end{array}$ & $17(3.1)$ & $59(3.8)$ & $109(2.7)$ \\
\hline $\begin{array}{c}\text { Not } \\
\text { documented }\end{array}$ & $12(2.9)$ & $5(1.0)$ & $4(0.6)$ & $\begin{array}{c}11 \\
(1.1) \\
\end{array}$ & $32(1.3)$ & $2(0.6)$ & $8(2.3)$ & $6(2.0)$ & $11(2.0)$ & $27(1.8)$ & $59(1.1)$ \\
\hline \multicolumn{12}{|c|}{ HIV Status } \\
\hline Positive & $41(11.9)$ & $\begin{array}{c}63 \\
(13.8) \\
\end{array}$ & $\begin{array}{c}89 \\
(14.3) \\
\end{array}$ & $\begin{array}{c}112 \\
(12.6) \\
\end{array}$ & $305(13.2)$ & $31(10.5)$ & $\begin{array}{c}52 \\
(16.1) \\
\end{array}$ & $\begin{array}{c}32 \\
(13.2) \\
\end{array}$ & $\begin{array}{c}65 \\
(15.4) \\
\end{array}$ & $179(14.0)$ & $484(13.5)$ \\
\hline Negative & $\begin{array}{c}304 \\
(88.1)\end{array}$ & $\begin{array}{c}393 \\
(86.2)\end{array}$ & $\begin{array}{c}533 \\
(85.7)\end{array}$ & $\begin{array}{c}779 \\
(87.4)\end{array}$ & $2009(86.8)$ & $\begin{array}{c}263 \\
(80.5)\end{array}$ & $\begin{array}{c}271 \\
(83.9)\end{array}$ & $\begin{array}{c}210 \\
(86.8)\end{array}$ & $\begin{array}{c}358 \\
(84.6)\end{array}$ & $1102(86.0)$ & $3111(86.5)$ \\
\hline $\begin{array}{c}\text { Not } \\
\text { documented }\end{array}$ & $62(15.2)$ & $35(7.1)$ & $58(8.5)$ & $\begin{array}{c}70 \\
(7.3) \\
\end{array}$ & 225 (8.9) & $\begin{array}{c}40 \\
(12.0) \\
\end{array}$ & $\begin{array}{c}27 \\
(8.2) \\
\end{array}$ & $\begin{array}{c}57 \\
(19.1) \\
\end{array}$ & $\begin{array}{c}125 \\
(22.8) \\
\end{array}$ & $252(16.4)$ & $477(11.7)$ \\
\hline \multicolumn{12}{|c|}{ ART Initiation } \\
\hline Yes & $29(72.5)$ & $\begin{array}{c}60 \\
(98.4)\end{array}$ & $\begin{array}{c}81 \\
(92.0)\end{array}$ & $\begin{array}{c}104 \\
(95.3)\end{array}$ & $274(92.0)$ & $\begin{array}{c}22 \\
(71.0)\end{array}$ & $\begin{array}{c}47 \\
(82.2)\end{array}$ & $\begin{array}{c}24 \\
(82.8)\end{array}$ & $\begin{array}{c}59 \\
(90.8)\end{array}$ & $152(86.4)$ & $426(89.9)$ \\
\hline No & $11(27.5)$ & $1(1.6)$ & $7(8.0)$ & $5(4.7)$ & $24(8.0)$ & $9(29.0)$ & $4(7.8)$ & $\begin{array}{c}5 \\
(17.2) \\
\end{array}$ & $6(9.2)$ & $24(13.6)$ & $48(10.1)$ \\
\hline
\end{tabular}

TB treatment outcomes (2014-2017)

\section{Treatment outcomes of all PTB patients}

The TSR (cured plus treatment completed) of the total 4,072 PTB patients registered in the four years was $80.7 \%$ (decreasing from $86.2 \%$ in 2015 to $76.2 \%$ in 2017), which is below the global target $(\geq 90 \%)$ as set by WHO End TB strategy. Other treatment outcomes were $8.6 \%$ lost to follow-up (LTFU), $5.8 \%$ not evaluated, $4.2 \%$ died, and $0.8 \%$ treatment failed. Overall, $554(13.6 \%)$ of the PTB patients had unsuccessful treatment outcome (treatment failed, died and LTFU) during the study period (increasing from 10.4\% in 2014 to $17.7 \%$ in 2017) which is a signal for MDR-TB (Table 3 ). LTFU rates (8.6\%) and death rates $(4.2 \%)$ were the major contributors for the unsuccessful treatment outcomes for PTB patients.
Treatment outcome of patient with bacteriologically confirmed PTB (BCPTB) and clinically diagnosed PTB (CDPTB)

As shown in Table 4, there was no significant difference in the pooled (2014-2017) TSR between BCPTB and CDPTB patients (80.2\% vs. $81.3 \%, \mathrm{P}=0.49)$. However, there was higher TSR in CDPTB than in BCPTB patients in 2014 (86.5\% vs. $81.3 \%)$ and 2015 (87.5\% vs. $84.8 \%)$, the same in 2016 (81.0 vs. $80.6 \%$ ); while it was higher in BCPTB than in CDPTB in 2017 (77.1\% vs. 74.3\%). The trend in TSR decreased across the years (2015 - 2017) for both BCPTB (84.8\% to $77.1 \%)$ and CDPTB (87.5\% to $74.3 \%)$ patients, which remained below the WHO End TB global target ( $\geq 90 \%)$ over the study period for both groups. Overall, the number of patients not evaluated was higher $(6.1 \%$ vs. $5.2 \%)$ in BCPTB than in CDPTB patients, whereas death rate was higher in CDPTB than in BCPTB (5.2\% vs. $3.6 \%)$.

Table 3: Trends of TB treatment outcomes for all pulmonary tuberculosis (PTB) patients registered for treatment in 67 refugee health facilities in IGAD region, 2014-2017 $(n=4072)$.

\begin{tabular}{|c|c|c|c|c|c|}
\hline Treatment outcomes & $\begin{array}{c}\text { 2014, }(\mathrm{n}=742) \\
\text { N (\%) }\end{array}$ & $\begin{array}{c}\text { 2015, }(\mathrm{n}=840) \\
\text { N (\%) }\end{array}$ & $\begin{array}{c}\text { 2016, }(\mathrm{n}=979) \\
\mathrm{N}(\%)\end{array}$ & $\begin{array}{c}2017,(n=1511) \\
\text { N (\%) }\end{array}$ & $\begin{array}{c}\text { Total, }(\mathrm{n}=4072) \\
\text { N (\%) }\end{array}$ \\
\hline Cured & $271(36.5)$ & $323(38.5)$ & $433(44.2)$ & $581(38.5)$ & $1608(39.5)$ \\
\hline Treatment completed & $349(47.0)$ & $401(47.7)$ & $357(36.5)$ & $569(37.7)$ & $1676(41.2)$ \\
\hline Successful outcome & $620(83.5)$ & $724(86.2)$ & $790(80.7)$ & $1150(76.2)$ & $3284(80.7)$ \\
\hline Treatment Failed & $4(0.5)$ & $7(0.8)$ & $6(0.6)$ & $15(1.0)$ & $32(0.8)$ \\
\hline LTFU & $47(6.3)$ & $44(5.2)$ & $76(7.8)$ & $185(12.2)$ & $352(8.6)$ \\
\hline Died & $27(3.6)$ & $33(3.9)$ & $42(4.3)$ & $68(4.5)$ & $170(4.2)$ \\
\hline Not evaluated & $44(5.9)$ & $32(3.8)$ & $65(6.6)$ & $93(6.2)$ & $234(5.8)$ \\
\hline Unsuccessful outcome & $78(10.4)$ & $84(9.9)$ & $124(12.7)$ & $268(17.7)$ & $554(13.6)$ \\
\hline
\end{tabular}

Unsuccessful treatment outcome is the sum of treatment failed, died and LTFU.

The pooled unsuccessful treatment outcome (treatment failed, died and LTFU) during the study period for both BCPTB and CDPTB was $13.6 \%$. The major contributor for the unsuccessful treatment for both BCPTB and CDPTB patients were LTFU (8.8\% and $8.2 \%)$ and died (3.6\% and 5.2\%) (Table 4). There was lower unsuccessful treatment outcome in CDPTB than in BCPTB patients in 2014 (7.2\% vs. 13.2\%) and 2015 (6.5\% vs. $11.1 \%)$, the same in 2016 (13.0 vs. $12.6 \%)$, while it was higher in CDPTB than in BCPTB in 2017 (20.8\% vs. 16.0\%) (Table 4). The trends in unsuccessful treatment outcome increased in both BCPTB (11.1\% to $16.0 \%)$ and in CDPTB (6.5\% to $20.8 \%)$ patients (Table 4, Figure 1).

\section{Factors affecting treatment outcomes}

\section{Treatment outcomes for CDPTB patients relative to BCPTB patients}

As shown in Table 5, treatment failure was $0.2 \%$ in CDPTB patients and $1.2 \%$ in BCPTB cases, and death rate was $5.1 \%$ in CDPTB patients and $3.6 \%$ in $\mathrm{BCPTB}$ cases. Controlling for age, gender and pretreatment weight $\leq 40 \mathrm{~kg}$, CDPTB patients were likely to die (Odds ratio [OR] OR 1.6, 95\% Confidence interval [CI]: 1.1-2.4, $P=0.01$ ) as compared to BCPTB patients 
Table 4: Trends of treatment outcomes of bacteriologically confirmed (BCPTB) and clinically diagnosed (CDPTB) pulmonary tuberculosis patients notified in 67 refugee health facilities in IGAD region from 2014 to $2017,(n=4072)$.

\begin{tabular}{|c|c|c|c|c|c|c|c|c|c|c|}
\hline \multirow{2}{*}{$\begin{array}{l}\text { Treatment } \\
\text { outcomes }\end{array}$} & \multicolumn{5}{|c|}{ BCPT $(\mathrm{N}=2539)$} & \multicolumn{5}{|c|}{ CDPT $(\mathrm{N}=1533)$} \\
\hline & $\begin{array}{c}2014 \\
(n=408)\end{array}$ & $\begin{array}{c}2015 \\
(n=489)\end{array}$ & $\begin{array}{c}2016 \\
(n=679)\end{array}$ & $\begin{array}{c}2017 \\
(\mathbf{n}=963)\end{array}$ & $\begin{array}{c}\text { Total } \\
(\mathbf{n}=2539)\end{array}$ & $\begin{array}{c}20.14 \\
(n=334)\end{array}$ & $\begin{array}{c}2015 \\
(\mathrm{n}=351)\end{array}$ & $\begin{array}{c}2016 \\
(n=300)\end{array}$ & $\begin{array}{c}2017 \\
(\mathbf{n}=548)\end{array}$ & $\begin{array}{c}\text { Total } \\
(\mathrm{n}=1533)\end{array}$ \\
\hline Successful & $331(81.3)$ & $417(84.8)$ & $547(80.6)$ & $743(77.1)$ & $2038(80.2)$ & $289(86.5)$ & $307(87.5)$ & $243(81.0)$ & $407(74.3)$ & $1246(81.3)$ \\
\hline Cured & $271(66.4)$ & $323(65.6)$ & $433(63.8)$ & $581(60.3)$ & $1608(63.3)$ & 0 & 0 & 0 & 0 & 0 \\
\hline \multirow[t]{2}{*}{ Completed } & $60(14.9)$ & $94(19.2)$ & $114(16.8)$ & $162(16.8)$ & $430(16.9)$ & $289(86.5)$ & 307 (87.5) & $243(81.0)$ & $407(74.3)$ & $1246(81.3)$ \\
\hline & $54(13.2)$ & $54(11.1)$ & $85(12.6)$ & $154(16.0)$ & $347(13.6)$ & $24(7.2$ & $30(6.5)$ & $39(13.0)$ & $114(20.8)$ & 207 (13.6) \\
\hline Failed & $4(1.0)$ & $6(1.2)$ & $5(0.7)$ & $14(1.5)$ & $29(1.1)$ & 0 & $1(0.3)$ & $1(0.3)$ & $1(0.2)$ & $3(0.2)$ \\
\hline LTFU & $36(8.8)$ & $33(6.8)$ & $53(7.8)$ & $105(10.9)$ & $227(8.9)$ & $11(3.3)$ & $11(3.1)$ & $23(7.7)$ & $80(14.6)$ & $125(8.2)$ \\
\hline Died & $14(3.4)$ & $15(3.1)$ & $27(4.0)$ & $35(3.6)$ & $91(3.6)$ & $13(3.9)$ & $18(5.1)$ & $15(5.0)$ & $33(6.0)$ & $79(5.2)$ \\
\hline $\begin{array}{c}\text { Not } \\
\text { evaluated }\end{array}$ & $23(5.6)$ & $18(3.7)$ & $47(7.0)$ & $66(6.9)$ & $154(6.1)$ & $21(6.3)$ & $14(4.0)$ & $18(6.0)$ & $27(4.9)$ & $80(5.2)$ \\
\hline
\end{tabular}

Data are presented as numbers (\%)

Unsuccessful: the sum of Failed, LTFU and Died treatment outcomes.

Table 5: Treatment outcomes of clinically diagnosed (CDPTB) relative to bacteriologically confirmed (BCPTB) PTB patients in 67 refugee health facilities in IGAD region from 2014 to $2017(\mathrm{n}=4,072)$.

\begin{tabular}{|c|c|c|c|c|c|c|c|}
\hline \multirow{2}{*}{ Treatment outcomes } & Total (N=4072) & BCPT (N=2539) & CDPT (N=1533) & \multicolumn{2}{|c|}{${ }^{*}$ Non-Adjusted } & \multicolumn{2}{|c|}{${ }^{* *}$ Adjusted } \\
\cline { 5 - 8 } & $\mathbf{N}(\%)$ & $\mathbf{N}(\%)$ & $\mathbf{N}(\%)$ & OR (95\% CI) & p-value & OR (95\% CI) & p-value \\
\hline Failed & $32(0.8)$ & $29(1.1)$ & $3(0.2)$ & $0.2(0.05-0.5)$ & 0.003 & $0.4(0.1-1.3)$ & 0.12 \\
\hline LTFU & $352(8.6)$ & $227(8.9)$ & $125(8.2)$ & $0.9(0.7-1.1)$ & 0.38 & $0.8(0.6-1.2)$ & 0.27 \\
\hline Died & $170(4.2)$ & $91(3.6)$ & $79(5.2)$ & $1.4(1.1-1.9)$ & 0.02 & $1.6(1.1-2.4)$ & 0.01 \\
\hline Not evaluated & $234(5.7)$ & $154(6.1)$ & $80(5.2)$ & $0.8(0.6-1.0)$ & 0.17 & $1.0(0.6-1.4)$ & 0.97 \\
\hline Unsuccessful & $554(13.6)$ & $347(13.6)$ & $207(13.6)$ & $0.9(0.6-1.1)$ & 0.26 & $0.9(0.6-1.1)$ & 0.4 \\
\hline
\end{tabular}

${ }^{*}$ Odds Ratio (OR) of treatment outcomes for clinically diagnosed PTB (CDPTB) patients relative to bacteriologically confirmed PTB (BCPTB) patients.

${ }^{* *}$ Adjusted for age, gender, and pre-treatment body weight,

Unsuccessful: the sum of Failed, LTFU and Died treatment outcomes.

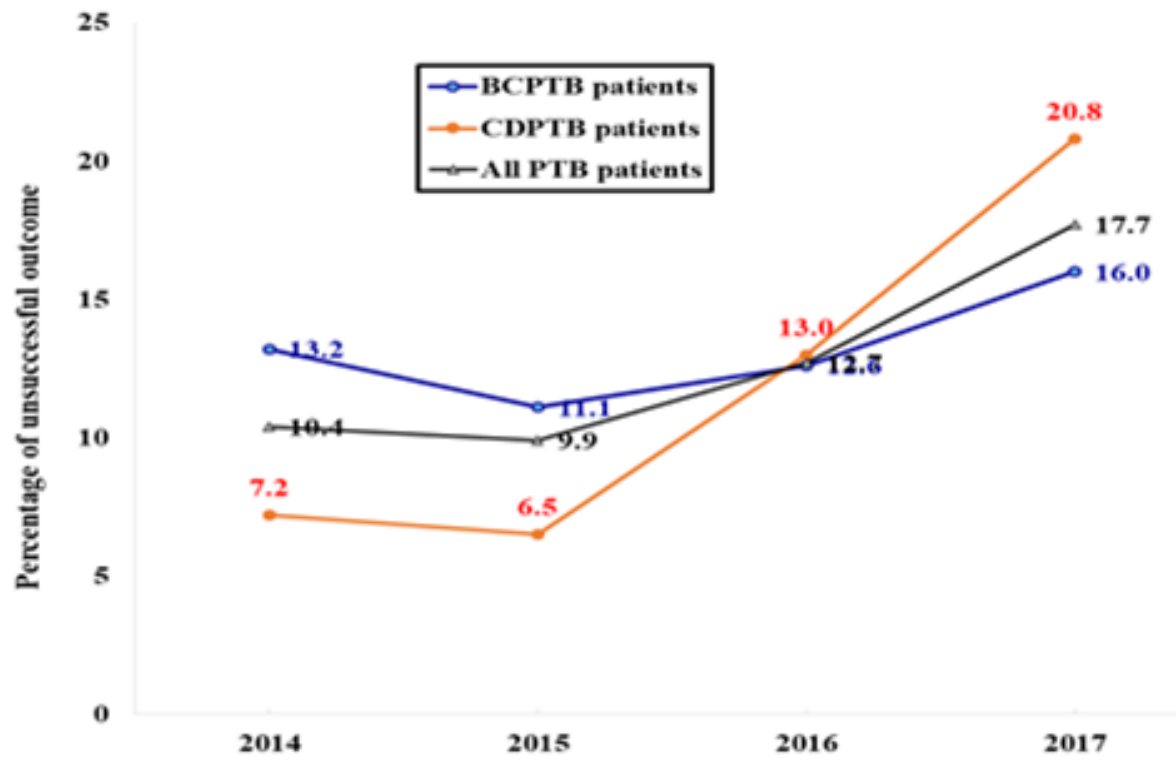

Figure 1: Trends of unsuccessful treatment outcome of all pulmonary tuberculosis (PTB) patients (black line), bacteriologically confirmed PTB (BCPTB) (blue line), and clinically diagnosed PTB (CDPTB) patients (red line) notified in 67 refugee health facilities in the IGAD region from 2014 to 2017 ( $\mathrm{n}=4,072$ ).

Factors associated with unsuccessful TB treatment outcomes among BCPTB and CDPTB patients

Table 6 \& Table 7 show factors associated with unsuccessful TB treatment outcomes among $\mathrm{BCPTB}$ and $\mathrm{CDPTB}$ patients, respectively.
Based on a multivariable logistic regression model, HIV infection was associated with unsuccessful TB treatment outcome among for both $\mathrm{BCPTB}(\mathrm{aOR}=2.6[95 \% \mathrm{CI}: 1.7-4.2])$ and CDPTB $(\mathrm{aOR}=2.8[95 \%$ CI: 2.0-3.8]) patients. 
On the other hand, being male $(\mathrm{aOR}=1.7$ [95\% CI: 1.4-3.8]) and having age 55-64 years (aOR=1.7, [95\% CI: $1.1-2.80]$ ) were associated with unsuccessful treatment outcomes among BCPTB patients (Table 6). Age groups $25-34$ years ( $\mathrm{aOR}=2.9$ [95\% CI: 1.6-5.2]), 35-44 years
$(\mathrm{aOR}=4.6[95 \% \mathrm{CI}: 2.6-8.3]), 65$ years and above $(\mathrm{aOR}=6.0[95 \%$ CI:3.1-11.5]) and having previous history of $\mathrm{TB}(\mathrm{aOR}=1.7[95 \%$ CI:1.0-2.7]) were associated with unsuccessful treatment outcomes among CDPTB patients (Table 7).

Table 6: Factors associated with unsuccessful treatment outcomes for bacteriologically confirmed pulmonary tuberculosis patients in 67 refugee health facilities, IGAD region, 2014-2017 $(\mathrm{n}=2,539)$.

\begin{tabular}{|c|c|c|c|c|c|c|c|}
\hline \multirow{2}{*}{ Characteristics } & \multicolumn{3}{|c|}{$\begin{array}{c}\text { Bivariate analysis } \\
\text { p-value }\end{array}$} & \multicolumn{2}{|c|}{ Univariate logistic analysis } & \multicolumn{2}{|c|}{ Multivariable logistic analysis } \\
\hline & $\begin{array}{l}\text { Successful } \\
\text { Outcomes }\end{array}$ & $\begin{array}{l}\text { Unsuccessful } \\
\text { Outcomes }\end{array}$ & p-Value & Unadjusted OR (95\%CI) & $P$ Value & $\begin{array}{c}\text { Adjusted OR } \\
(95 \% \mathrm{CI})\end{array}$ & P Value \\
\hline \multicolumn{8}{|c|}{ Gender } \\
\hline Female & $771(87.2)$ & $113(12.8)$ & 0.05 & 1 & & & \\
\hline Male & $1233(84.3)$ & $233(15.7)$ & & $1.3(1.0-1.6)$ & 0.05 & $1.7(1.4-3.8)$ & 0.001 \\
\hline \multicolumn{8}{|c|}{ Age Group } \\
\hline$<15$ & $100(92.5)$ & $8(7.5)$ & & 1 & & 1 & \\
\hline $15-24$ & $524(88.8)$ & $66(11.2)$ & & $1.6(0.7-3.4)$ & 0.2 & - & - \\
\hline 25-34 & $616(89.7)$ & $71(10.3)$ & $<0.001$ & $1.4(0.7-3.1)$ & 0.3 & - & - \\
\hline $35-44$ & $352(81.5)$ & $80(18.5)$ & & $2.9(1.3-6.1)$ & 0.007 & - & - \\
\hline 45-54 & $226(82.5)$ & $48(17.5)$ & & $2.7(1.2-5.9)$ & 0.01 & $1.0(0.6-1.6)$ & 0.97 \\
\hline $55-64$ & $122(72.2)$ & $47(27.8)$ & & $4.9(2.2-10.8)$ & $<0.001$ & $1.7(1.1-2.8)$ & 0.02 \\
\hline$>=65$ & $87(77.7)$ & $25(22.3)$ & & $3.7(1.6-8.6)$ & 0.003 & $1.5(0.9-2.6)$ & 0.16 \\
\hline \multicolumn{8}{|c|}{ Patient category } \\
\hline New & $1852(86.6)$ & $287(13.4)$ & $<0.001$ & 1 & & & \\
\hline Retreatment ${ }^{* *}$ & $130(74.3)$ & $45(25.7)$ & & $2.2(1.6-3.2)$ & $<0.001$ & $1.5(1.0-2.2)$ & 0.06 \\
\hline \multicolumn{8}{|c|}{ Pre-Treatment Weight, $\mathrm{kg}^{\star}$} \\
\hline$>40$ & $1207(89.4)$ & $143(10.6)$ & 0.001 & 1 & & & \\
\hline$\leq 40$ & $321(83.4)$ & $64(16.6)$ & & $1.7(1.2-2.3)$ & 0.002 & $1.6(0.8-2.9)$ & 0.12 \\
\hline \multicolumn{8}{|c|}{ HIV Status } \\
\hline Negative & $1686(87.5)$ & $240(12.5)$ & $<0.001$ & 1 & & & \\
\hline Positive & $217(77.8)$ & $62(22.2)$ & & $2.0(1.5-2.7)$ & $<0.001$ & $2.6(1.7-4.2)$ & $<0.001$ \\
\hline \multicolumn{8}{|c|}{ ART initiated } \\
\hline Yes & $202(78.9)$ & $54(21.1)$ & 0.4 & 1 & 0.4 & & \\
\hline No & $50(83.3)$ & $10(16.7)$ & & $0.7(0.3-1.5)$ & & & \\
\hline
\end{tabular}

${ }^{*}$ Retreatment includes relapse, Treatment after failure, Treatment after loss to follow-up, and other previously treated patients; ${ }^{*}$ Analysis done for adults aged $\geq$ 15 years old.

Table 7: Factors associated with unsuccessful treatment outcomes for clinically diagnosed pulmonary tuberculosis patients in 67 refugee health facilities, IGAD region, 2014-2017 $(\mathrm{n}=1,533)$.

\begin{tabular}{|c|c|c|c|c|c|c|c|}
\hline \multirow{2}{*}{ Characteristics } & \multicolumn{3}{|c|}{ Bivariate Analysis } & \multicolumn{2}{|c|}{ Univariate Logistic Analysis } & \multicolumn{2}{|c|}{$\begin{array}{c}\text { Multivariable Logistic } \\
\text { Analysis }\end{array}$} \\
\hline & $\begin{array}{l}\text { Successful } \\
\text { Outcomes }\end{array}$ & $\begin{array}{l}\text { Unsuccessful } \\
\text { Outcomes }\end{array}$ & p-Value & $\begin{array}{c}\text { Unadjusted OR } \\
(95 \% \mathrm{CI})\end{array}$ & P Value & $\begin{array}{l}\text { Adjusted OR } \\
(95 \% \mathrm{CI})\end{array}$ & P Value \\
\hline \multicolumn{8}{|c|}{ Gender } \\
\hline Female & $572(86.8)$ & $87(13.2)$ & 0.3 & 1 & & & \\
\hline Male & $657(84.9)$ & $117(15.1)$ & & $1.2(0.9-1.6)$ & 0.3 & & \\
\hline \multicolumn{8}{|c|}{ Age Group } \\
\hline$<15$ & $479(90.4)$ & $51(9.6)$ & & 1 & & & \\
\hline $15-24$ & $141(87.0)$ & $21(13.0)$ & 0.007 & $1.4(0.8-2.4)$ & 0.2 & & \\
\hline $25-34$ & $187(81.7)$ & $42(18.3)$ & & $2.0(1.3-3.1)$ & 0.001 & $2.9(1.6-5.2)$ & $<0.001$ \\
\hline $35-44$ & $145(81.5)$ & $33(18.5)$ & & $2.1(1.3-3.3)$ & 0.002 & $4.6(2.6-8.3)$ & $<0.001$ \\
\hline $45-54$ & $113(84.3)$ & $21(15.7)$ & & $1.7(1.0-2.8)$ & 0.05 & & \\
\hline $55-64$ & $84(85.7)$ & $14(14.3)$ & & $1.6(0.8-2.9)$ & 0.2 & & \\
\hline
\end{tabular}




\begin{tabular}{|c|c|c|c|c|c|c|c|}
\hline$>=65$ & $91(79.8)$ & $23(20.2)$ & & $2.4(1.3-4.0)$ & 0.002 & $6.0(3.1-11.5)$ & $<0.001$ \\
\hline \multicolumn{8}{|c|}{ Patient Category } \\
\hline New & $1141(87.1)$ & $169(12.9)$ & 0.05 & 1 & & & \\
\hline Retreatment ${ }^{* *}$ & $52(78.8)$ & $14(21.2)$ & & $1.8(1.0-3.2)$ & 0.05 & $1.7(1.0-2.7)$ & 0.03 \\
\hline \multicolumn{8}{|c|}{ Pre-Treatment Weight, kg * } \\
\hline$>40$ & $471(86.7)$ & $72(13.3)$ & 0.3 & 1 & & & \\
\hline$\leq 40$ & $195(84.0)$ & $37(16.0)$ & & $1.3(0.8-2.0)$ & 0.3 & & \\
\hline \multicolumn{8}{|c|}{ HIV Status } \\
\hline Negative & $964(90.7)$ & $99(9.3)$ & $<0.001$ & 1 & & & \\
\hline Positive & $119(71.7)$ & $47(28.3)$ & & $3.8(2.5-5.6)$ & $<0.001$ & $2.8(2.0-3.8)$ & $<0.001$ \\
\hline \multicolumn{8}{|c|}{ ART Initiated } \\
\hline Yes & $105(72.9)$ & $39(27.1)$ & 0.2 & 1 & 0.3 & & \\
\hline No & $71(79.8)$ & $18(20.2)$ & & $0.7(0.4-1.3)$ & & & \\
\hline
\end{tabular}

${ }^{\star}$ Retreatment includes relapse, Treatment after failure, Treatment after loss to follow-up, and other previously treated patients; ${ }^{\star}$ Analysis done for adults aged $\geq$ 15 years old.

\section{Discussion}

The WHO End TB strategy indicates that refugees are among the key population for TB and at least $90 \%$ of them need to be reached with TB services by 2020. To ensure this, continuous monitoring of the TB program performance in the refugee settings is paramount $[3,13,15]$. The TSR of PTB patients over the four-year period ranged from $76.2 \%$ to $86.2 \%$ (Table 3), which is higher than that reported in refugee camps in different parts of the world (ranging from $63.6 \%$ to 77.5\%) [30-35] but way below the set End TB global target ( $\geq 90 \%$ ), [3]. Comparing the TSR achieved in the refugee camps to national TSR (for new and relapse TB cases) in the refugee hosting countries for year 2017 [36], Ethiopia (74.3\% vs. $96.0 \%)$, Sudan (69.0\% vs. $78 \%)$ and Uganda (71.6\% vs. $77 \%)$ all had lower TSR. This was however, similar in Kenya (93.6\% vs. 94.1). The low TSR in the refugee camps could be related to the limited evaluation of treatment outcomes in the refugee camps with limited resources [22], presence of other risk factors that affect the outcome of TB treatment, or due to less performance of TB program in the refugee camps $[8,9]$. Together, our findings suggest the need to improve the TB treatment program in the refugee camps via strengthening collaboration and coordination among partners working on TB, particularly with Primary health care providers in the refugee settings, TB program of local Health authorities and the NTP [37]. The high and increasing trend in unsuccessful treatment outcomes depicted a signal for MDR-TB. This however, was comparably lower than the $25.8 \%$ in refugee communities in Gambella, Southwest Ethiopia [8], but higher than the $14.8 \%$ reported in Southern Ethiopia [38].

In the refugee camps, LTFU outcome was on the rise and was a major contributor of the unsuccessful outcome. The reported LTFU in 2017 was lower than that reported in refugee camps in Gambella, Ethiopia (18.5\%) [8] and in Thai-Burmese border, Thailand (13.5\%) [34], but higher than that reported in Ngara, Tanzania (7.1\%) [35]. The pattern could be related to inadequate health education, suboptimal strategies to trace lost to treatment follow up patients, transfer of patients not done correctly, movements into and out of the camps as a result of instability or inconvenient time and staff handling of the patients. Similar to LTFU, death rate while being the second largest contributor to the unsuccessful TB treatment outcome witnessed an upward trend. This was lower than the death rates reported in [8] (6.5\%), [34] (7.5\%) and [35] (10.9\%). The could be attributed to late diagnosis and TBHIV co-infection.

\section{Treatment outcome by diagnostic category}

The priority of TB programs is to identify and treat BCPTB patients who are the source of TB transmission to the community. Therefore, treatment outcome performance among the BCPTB patients is an important guide assessing quality of a TB program [15]. On the other hand, the perception of CDPTB as less infectious in the community has led to their minimal support and monitoring $[23,24]$. This is despite evidence indicating that CDPTB patients contributed up to $17 \%$ of TB transmission [40]. The declining trend in CDPTB patients across the study period could be as a result of preference of laboratory evidence among adults, increased availability of gene xpert machines which have been shown to be more superior to (Acid- Fast Bacilli) AFB microscopy. This notwithstanding, more attention should be given to CDPTB patients in the refugee camps to improve the overall TB control activities.

In addition to the low case finding among CDPTB patients, their lower TB treatment outcome as compared to BCPTB could have further been spiraled by their delayed health care seeking behavior and TBHIV co-infections [23,24]. Nonetheless, WHO [41], recommends similar treatment (regimen, dosage, dosing frequency and duration) for all drug susceptible patients. In contrast to our findings, higher TSR than that of CDPTB but similar to that of BCPTB patients has been reported [42]. Different studies $[8,9,34,35]$ have related the varying TSRs to the difference in clinical (HIV status, TB patient category) and demographic (age, gender) characteristic of the patients, and other site-specific risk factors which are known to affect treatment success.

The unsuccessful treatment outcome was similar between BCPTB and CDPTB patients during the study period. Also, there was no difference in TB-HIV coinfection between BCPTB and CDPTB. This was inconsistent with findings by [42] which showed higher unsuccessful treatment in smear negative than smear positive PTB (25.3\% vs. $9.7 \%)$ and higher HIV co-infection in smear negative than smear positive PTB (41.4\% vs. 13.5\%). Compared to CDPTB, patients with BCPTB had higher LTFU and higher proportion of not evaluated patients, consistent findings with [24]. The findings by [42], however, showed higher failure and LTFU in smear negative than smear positive PTB.

The high death rate noted among CDPTB than in BCPTB was similar to findings by $[24,42]$. The higher death rates in CDPTB patients could be due to advanced disease stage linked to higher HIV infection, late diagnosis, delayed access to the health care services, and lack of support 
given to the patients $[23,24]$. Identification of factors associated with unsuccessful TB treatment outcomes is essential for improved TSR and reduced morbidity and mortality. Old age and HIV infection were significantly associated with unsuccessful TB treatment outcome among patients with BCPTB as well as CDPTB. These were consistent to $[8,34,35]$. The possible reasons for unsuccessful treatment outcome in older age groups include high likelihood to experience LTFU or death [42-44], atypical clinical presentation which affect TB diagnosis and may lead to increased mortality $[44,45]$, or the increased tendency to non-adherence to TB treatment by the older age group population possibly because of tight work schedule, long distance travel in search for work and alcoholism [35, 43-45].

TB-HIV co-infected patients were more likely to have unsuccessful treatment outcome in BCPTB and CDPTB, comparable findings to $[8,43-45]$. This could be due to non-adherence which may lead to drug burden, overlapping drug toxicities, or less drug absorption relating to drug-drug interaction $[43,44]$. Specific association with unsuccessful outcome among patients with BCPTB was observed among male gender and with CDPTB among previously treated TB patients. The former risk was consistent with findings in $[8,47-49]$ while the latter risk agreed with $[8,32]$. This could be related to misclassification of patient category or choice of wrong regimen or drug quality among previously treated and higher social interaction outside home, mobility from place to place by male population. Pre-treatment weight $\leq 40 \mathrm{~kg}$ was associated with unsuccessful treatment among BCPTB patients, which was consistent to findings by [46].

The results identified four-year pooled TB treatment outcome and important relationship between the baseline covariates and treatment outcomes among PTB patients. These findings were based on secondary data from routine surveillance. Limitations could therefore, include the usual operational problems of working with routine programmatic records that may have inaccuracies and inconsistencies.

The strengths of this study include four-year pooled TB treatment outcome and identified factors associated with unsuccessful treatment outcomes among BCPTB and CDPTB patients. usual operational problems of working with routine programmatic records that may have inaccuracies and inconsistencies. Lastly, future studies to consider additional factors that have been shown to be associated with unsuccessful TB treatment outcome such as occupation and education level $[8,50]$, smoking and diabetes $[51,52]$, and TB drug resistance status [8].

\section{Conclusion}

From the findings, we conclude that TB Treatment success rate in the refugee camps remained below the global target $(\geq 90)$. The major contributors of the low performance were LTFU and death rate. The factors associated with unsuccessful TB treatment outcome was HIV infection for both BCPTB and СDPTB patients; being male and age 55-64 years among BCРТB patients; and being retreatment TB patient, age $25-34,35-44$ years and $\geq 65$ years among CDPTB patients. These populations should be paid attention and monitored intensively in the refugee camps while seeing $\mathrm{TB}$ patients so as to improve the TB treatment outcomes.

\section{References}

1. Global tuberculosis report 2020. World Health Organization 2020. Geneva: USA.

2. World Health Organization (WHO). The End TB Strategy. World Health Organization 2015. Geneva: USA.

3. Kimbrough W, Saliba V, Dahab M, Haskew C, Checchi F (2012) The burden of tuberculosis in crisis-affected populations: a systematic review. Lancet Infect Dis 12(12): 950-965.
4. WHO (2016) Interregional workshop for tuberculosis control and care among refugees and migrant populations, 10-11 May 2016. Catania, Italy.

5. Nuzzo JB, Golub JE, Chaulk P, Shah M (2015) Postarrival Tuberculosis Screening of High-Risk Immigrants at a Local Health Department. Am J Public Health 105(7): 1432-1438.

6. Greenaway C, Sandoe A, Vissandjee B, Kitai I, Gruner D, et al. (2011) Tuberculosis: evidence review for newly arriving immigrants and refugees. CMAJ 183(12): E939-E951.

7. Acosta CD, Kaluski DN, Dara M (2014) Conflict and drug-resistant tuberculosis in Ukraine. Lancet 384(9953): 1500-1501.

8. Ejeta E, Beyene G, Balay G, Bonsa Z, Abebe G (2018) Factors associated with unsuccessful treatment outcome in tuberculosis patients among refugees and their surrounding communities in Gambella Regional State, Ethiopia. PLoS ONE 13(10): e0205468.

9. Dogru S, Doner P (2017) Frequency and outcomes of new patients with pulmonary tuberculosis in Hatay province after Syrian civil war. Indian J Tuberc 64(2): 83-88.

10. World Health Organization (WHO). Interregional workshop for tuberculosis control and care among refugees and migrant populations. Catania: Italy; 2016.

11. World Health Organization. Stop TB strategy. World Health Organization 2006. Geneva: USA.

12. World Health Organization. Tuberculosis control in refugee situations: An Inter-agency field manual. (WHO/TB/97.221) 1997.

13. United Nations High Commissioner For Refugees (UNHCR). Global Trends: Forced Displacement in 2018. UNHCR; Geneva: USA; 2019.

14. Njuki C, Abera W (2018) Forced displacement and mixed migration challenges in the IGAD region. GREAT insights Magazine 7(1).

15. World Health Organization. Compendium of WHO guidelines and associated standards: ensuring optimum delivery of the cascade of care for patients with tuberculosis, second edition. Geneva: USA; 2018.

16. World Health Organization. Treatment of tuberculosis: guidelines. 2010

17. Shingadia D, Novelli V (2003) Diagnosis and treatment of tuberculosis in children. Lancet Infect Dis 3(10):624-632.

18. Dogru S, Doner P (2017) Frequency and outcomes of new patients with pulmonary tuberculosis in Hatay province after Syrian civil war. Indian J Tuberc 64(2): 83-88.

19. Sukrakanchana-Trikham P, Puechal X, Rigal J, Rieder HL (1992) 10 year assessment of treatment outcome among Cambodian refugees with sputum smear-positive tuberculosis in Khao-l-Dang, Thailand. Tuber Lung Dis 73(6): 384-387.

20. Minetti A, Camelique O, Hsa Thaw K, Thi S, Swaddiwudhipong W, et al. (2010) Tuberculosis treatment in a refugee and migrant population: 20 years of experience on the Thai-Burmese border. Int J Tuberc Lung Dis 14(12): 1589-1595.

21. Rutta E, Kipingili R, Lukonge H, Assefa S, Mitsilale E, et al. (2001) Treatment outcome among Rwandan and Burundian refugees with sputum smear-positive tuberculosis in Ngara, Tanzania. Int J Tuberc Lung Dis 5(7): 628-632

22. World Health Organization. Global Health Observatory data repository [Internet]. Treatment coverage Data by WHO region. 2019

23. Hargreaves NJ, Kadzakumanja O, Phiri S, Nyangulu DS, Salaniponi FM, et al. (2001) What causes smear-negative pulmonary tuberculosis in Malawi, an area of high HIV seroprevalence? Int J Tuberc Lung Dis 5(2): 113-122.

24. Harries AD, Nyirenda TE, Banerjee A, Boeree MJ, Salaniponi FM (1999) Treatment outcome of patients with smear-negative and smearpositive pulmonary tuberculosis in the National Tuberculosis Control Programme, Malawi. Trans R Soc Trop Med Hyg 93(4): 443-446.

25. Federal Ministry of Health $(\mathrm{FMOH}) .6^{\text {th }}$ ed. National guidelines for TB, DR-TB and Leprosy in Ethiopia. 2017 
26. Ministry of Health (MOH), Uganda. Manual for management and control of Tuberculosis and Leprosy in Uganda, $3^{\text {rd }}$ ed. (MOH, Uganda, 2017).

27. Ministry of Health (MOH). National TB Management Guideline ( $\mathrm{MOH}$, Sudan, 2018).

28. Ministry of Health, republic of Kenya. Guideline for integrated Tuberculosis, leprosy and lung disease in Kenya, September 2017.

29. World Health Organization. Definitions and reporting framework for tuberculosis-2013 revision. WHO; Geneva: USA; 2013.

30. Shingadia D, Novelli V (2003) Diagnosis and treatment of tuberculosis in children. Lancet Infect Dis 3(10): 624-632.

31. Dogru S, Doner P (2017) Frequency and outcomes of new patients with pulmonary tuberculosis in Hatay province after Syrian civil war. Indian J Tuberc 64(2): 83-88.

32. Vo LNQ, Codlin AJ, Forse RJ, Nguyen HT, Vu TN, et al. (2020) Tuberculosis among economic migrants: across-sectional study of the risk of poor treatment outcomes and impact of a treatment adherence intervention among temporary residents in an urban district in Ho Chi Minh City, Viet Nam. BMC Infect Dis 20(1): 134.

33. Sukrakanchana-Trikham P, Puéchal X, Rigal J, Rieder HL (1992) 10year assessment of treatment outcome among Cambodian refugees with sputum smear-positive tuberculosis in Khao-1-Dang, Thailand. Tuber Lung Dis 73(6): 384-387

34. Minetti A, Camelique O, Hsa Thaw K, Thi S, Swaddiwudhipong W, et al (2010) Tuberculosis treatment in a refugee and migrant population: 20 years of experience on the Thai-Burmese border. Int J Tuberc Lung Dis 14(12): 1589-1595.

35. Rutta E, Kipingili R, Lukonge H, Assefa S, Mitsilale E, et al. (2001) Treatment outcome among Rwandan and Burundian refugees with sputum smear-positivetuberculosis in Ngara, Tanzania. Int J Tuberc Lung Dis 5(7): 628-632.

36. Global tuberculosis report 2018. World Health Organization; Geneva: USA; 2018

37. Porter J, Kessler C (1995) Tuberculosis in refugees: a neglected dimension of the global epidemic of tuberculosis. Trans Roy Soc Trop Med Hygiene 89(3): 241-242.

38. Gebrezgabiher G, Romha G, Ejeta E, Asebe G, Zemene E, et al. (2016) Treatment outcome of tuberculosis patients under directly observed treatment short course and factors affecting outcome in southern Ethiopia: a five-year retrospective study. PLoS One 11(2): e0150560.

39. Amante TD, Ahemed TA (2015) Risk factors for unsuccessful tuberculosis treatment outcome (failure, default and death) in public health institutions, eastern Ethiopia. Pan Afr Med J 20: 247.
40. Behr MA, Warren SA, Salamon H, Hopewell PC, Leon APD, et al. (1999) Transmission of mycobacterium tuberculosis from patients smear negative for acid-fast bacilli. Lancet 353(9151): 444-449.

41. Guidelines for treatment of drug-susceptible tuberculosis and patient care, 2017 update. World Health Organization; 2017. Geneva: USA.

42. Adea S, Harries A D, Trébucq A, Hinderaker SG, Adé G, et al. (2013) National profile and treatment outcomes of adult smear-negative pulmonary TB patients in Benin. Trans R Soc Trop Med Hyg 107(12): 783-788.

43. Wen Y, Zhang Z, Li X, Xia D, Ma J, et al. (2018) Treatment outcomes and factors affecting unsuccessful outcome among new pulmonary smear positive and negative tuberculosis patients in Anqing, China: a retrospective study. BMC Infect Dis 18: 104.

44. Rajagopalan S (2001) Tuberculosis and aging: a global health problem. Clin Infect Dis 33(7): 1034-1039.

45. Tadesse F (2015) Risk factors for multi-drug resistant tuberculosis in Addis Ababa, Ethiopia. Universal Journal of Public Health 3(2): 65-70.

46. Velavan A, Purty AJ, Shringarpure K, Sagili KD, Mishra AK, et al. (2018) Tuberculosis retreatment outcomes and associated factors: a mixedmethods study from Puducherry, India. Public Health Action 8(4): 187193.

47. Melese A, Zeleke B, Ewnete B (2016) Treatment Outcome and Associated Factors among Tuberculosis Patients in Debre Tabor, Northwestern Ethiopia: A Retrospective Study. Tuberc Res Treat 2016: 1354356.

48. Muñoz-Sellart M, Cuevas LE, Tumato M, Merid Y, Yassin A (2010) Factors associated with poor tuberculosis treatment outcome in the Southern Regional of Ethiopia. Int J Tuberc Lung Dis 14(8): 973-979.

49. Ahmad T, Jadoon MA, Khan M, Haroon, Khan MM, Hussain A, et al (2020) Treatment outcomes of patients with tuberculosis in war affected region of Khyber Pakhtunkhwa, Pakistan. BMC Infectious Diseases 20(1): 463.

50. Dangisso MH, Datiko DG, Lindtiørn B (2015) Trends of Tuberculosis Case Notification and Treatment Outcomes in the Sidama Zone, Southern Ethiopia: Ten-Year Retrospective Trend Analysis in Urban-Rural Settings. PLoS ONE 10(4): e0125135.

51. Masjedi MR, Hosseini M, Aryanpur M, Mortaz E, Tabarsi P, et al. (2017) The effects of smoking on treatment outcome in patients newly diagnosed with pulmonary tuberculosis. Int J Tuberc Lung Dis 21(3): 351-356.

52. Jiménez-Corona ME, Cruz-Hervert LP, García-García L, FerreyraReyes L, Delgado-Sánchez G, et al. (2013) Association of diabetes and tuberculosis: impact on treatment and post-treatment outcomes. Thorax 68(3): 214-220 